\title{
Chiari network and patent foramen ovale associated with stroke
}

\author{
Adwaiy Manerikar, BA, and S. Chris Malaisrie, MD, Chicago, Ill
}

\footnotetext{
From the Division of Cardiac Surgery, Northwestern University Feinberg School of Medicine, Northwestern Medicine, Chicago, Ill.

Disclosures: S.C. Malaisrie reported Edwards: speaker and consultant; Abbott: speaker; Cryolife: consultant, and Terumo: speaker. A. Manerikar reported no conflicts of interest.

The Journal policy requires editors and reviewers to disclose conflicts of interest and to decline handling or reviewing manuscripts for which they may have a conflict of interest. The editors and reviewers of this article have no conflicts of interest.

Received for publication Nov 5, 2021; accepted for publication Nov 5, 2021; available ahead of print Nov 14, 2021.

Address for reprints: S. Chris Malaisrie, MD, 676 North St Clair, Arkes Pavilion 730, Chicago, IL 60611 (E-mail: chris.malaisrie@nm.org).

JTCVS Techniques 2022;11:45-7

2666-2507

Copyright $(2021$ The Author(s). Published by Elsevier Inc. on behalf of The American Association for Thoracic Surgery. This is an open access article under the CC BY-NC-ND license (http://creativecommons.org/licenses/bync-nd/4.0/).

https://doi.org/10.1016/j.xjtc.2021.11.006
}

Video clip is available online.

The Chiari network is a mesh-like fenestrated embryonic remnant that is found in the right atrium at the junction of the superior and inferior vena cavae. Although this finding is generally benign, it has been shown to favor the retention of a patent foramen ovale (PFO) and promote thrombotic and embolic complications. ${ }^{1}$ We present a 46-year-old man with previous history of cardioembolic stroke who was found to have Chiari network, concomitant PFO, and associated mitral valve mass. He underwent surgical excision through a minimally invasive open-heart surgery via mini right thoracotomy, and his postoperative course was uncomplicated. Northwestern University institutional review board reviewed the study (STU00214992) in May 2021, and determined the project was not research and no institutional review board approval is required. Institutional consent for use of de-identified information was obtained.

\section{CASE}

A 46-year-old man suffered a right occipital stroke with persistent hemianopsia. Transesophageal echocardiogram showed a $3.5-\mathrm{cm}$ mass in the right atrium, suggestive of an atypical Chiari network, a PFO, and a $1.3-\mathrm{cm}$ mitral valve mass consistent with a Lambl excrescence. There was trivial mitral regurgitation. Because the patient's Risk of Paradoxical Embolism score suggested a $72 \%$ chance

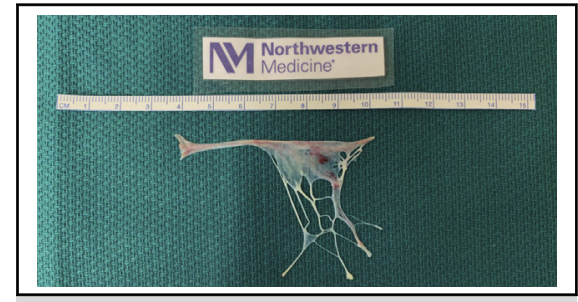

Chiari network after resection from right atrium.

CENTRAL MESSAGE

The Chiari network might pro-

mote patent foramen ovale fo-

ramen and result in stroke.

Successful removal might be

possible through a minimally

invasive open-heart approach.

See Commentary on page 48.

that the stroke was due to PFO, this prompted the decision to pursue closure. Furthermore, because Lambl excrescence and PFO are both known causes of stroke and the patient's clinical history did not indicate which could be the more likely cause, open-heart surgery for mitral valve mass resection, right atrial mass resection, and PFO closure was determined to be the optimal treatment for eliminating the likely causes of recurrent stroke (Video 1). The decision to pursue surgery over medical management or device closure of PFO was made by a multidisciplinary heart team consisting of

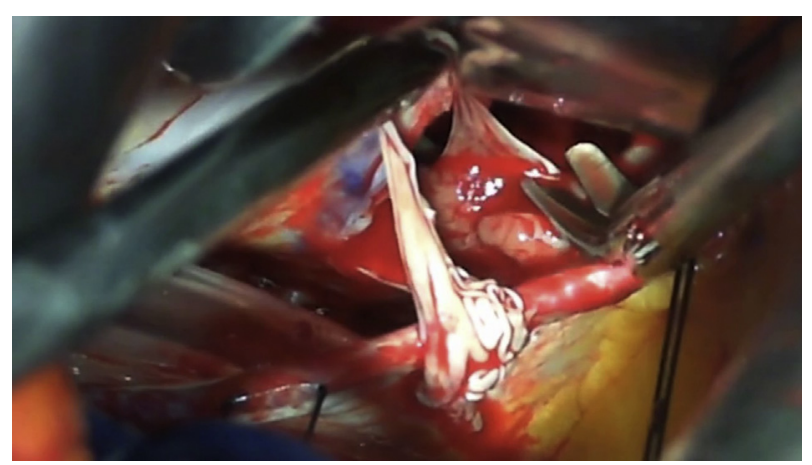

VIDEO 1. Chiari network and patent foramen ovale case study. A narrated case study with surgical video is presented. Video available at: https:// www.jtcvs.org/article/S2666-2507(21)00762-8/fulltext. 
cardiologists and cardiovascular surgeons. There was no attempt at anticoagulation before surgery, and antiplatelet therapy was halted 1 week before the operation.

After induction of anesthesia and establishment of arterial and venous access, the right chest wall was entered through the fourth intercostal space lateral to the right nipple. A trocar was placed at the sixth interspace for liver retraction and insufflation of $\mathrm{CO}_{2}$. Cardiopulmonary bypass was initiated with good venous drainage using femoral and superior vena cava cannulas. The pericardium was opened horizontally away from the phrenic nerve, and antegrade cold blood hyperkalemic cardioplegia was used. Left atriotomy was made, and an atrial lift retractor was placed through a stab incision through the fourth intercostal space just lateral to the sternal edge. The mitral valve was inspected and no mass was found, confirming findings on the intraoperative transesophageal echocardiogram. The PFO was then closed with 2 rows of running 4-0 polypropylene suture from the left atrial side. There was no associated septal aneurysm. The left atrium was closed with a single row of running 3-0 polypropylene suture and the heart was deaired through the anastomotic line. Crossclamp was then removed and the heart regained sinus rhythm spontaneously. A vertical right atriotomy was made in the lower third of the right atrium, and a large fenestrated membrane measuring $6 \mathrm{~cm}$ was resected from the area of the inferior vena cava (Figure 1). This structure was consistent with Chiari network (Figure 2). Another row of running 4-0 polypropylene suture was placed at the PFO on the right atrial side, and right atriotomy was closed with 2 rows of running 4-0 polypropylene suture. The patient was then weaned from bypass and decannulated. The patient's postoperative recovery was uncomplicated.

\section{DISCUSSION}

The Chiari network is the remnant of the embryologic right valve of sinus venosus, which normally regresses to form the crista terminalis and the eustachian and thebesian valves. Failure of regression leads to a web-like structure as shown in Figure 1. Although often an incidental finding, the Chiari network been shown to be associated with PFO and

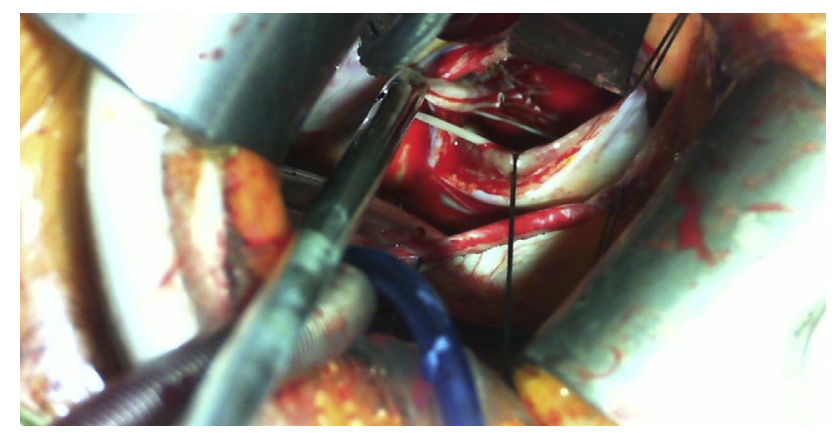

FIGURE 1. Intraoperative image of Chiari network dissection and removal from the right atrium.

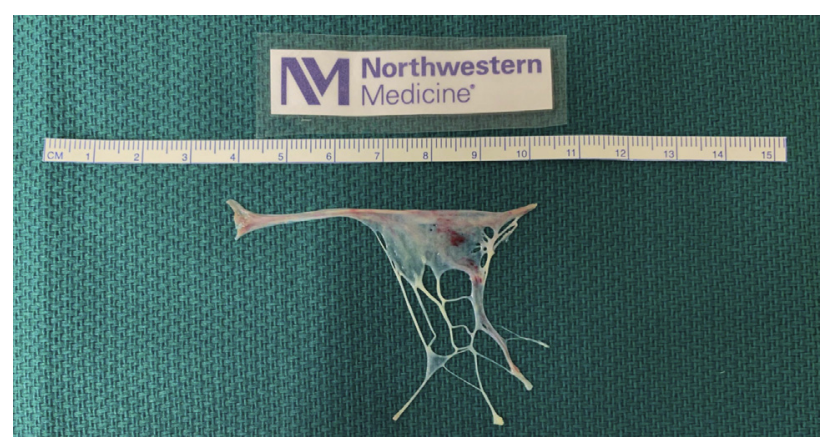

FIGURE 2. Postoperative image after resection of Chiari network measuring $6 \mathrm{~cm}$ in length.

atrial septal aneurysm. The proposed mechanism for this phenomenon is that the Chiari network results in an embryonic right atrial flow pattern, which directs blood toward the interatrial septum, leading to an increased likelihood of aneurysm or PFO development. ${ }^{1}$ Furthermore, the Chiari network has also been posited as a source of thrombi and emboli. ${ }^{2,3}$ Therefore, the combined presence of PFO with Chiari network suggests an individual's risk of developing cerebrovascular accident or transient ischemic attack is increased. This has been described in other reports in which the Chiari network has been associated with cerebrovascular complications. ${ }^{4}$ In our patient, the Risk of Paradoxical Embolism score suggested a strong likelihood that stroke was due to PFO, and provides further supporting evidence for Chiari network as a potential source of embolism. Furthermore, the Chiari network has also been associated with other complications such as valvular dysfunction, endocarditis, arrhythmia, and catheter-trapping. ${ }^{5}$ From these examples, it appears that although Chiari network is largely uncomplicated, it might not be as clinically benign as previously thought.

In this report, we describe a successful resection of Chiari network and simultaneous repair of PFO via minimally invasive cardiac surgery using mini right thoracotomy. Resection of the Chiari network should include the attachments to the os of the coronary sinus and the right atrial wall, while avoiding injury to the interatrial septum. We believe this is the first published instance of Chiari network removal using this approach.

Our report suggests that surgical removal of the Chiari network is possible using a minimally invasive cardiac approach. In addition, because the Chiari network might be associated with a wide variety of harmful consequences, including stroke and transient ischemic attack, surgical resection might prove to be a viable and justifiable option for management of this unique phenomenon.

\section{References}

1. Schneider B, Hofmann T, Justen MH, Meinertz T. Chiari's network: normal anatomic variant or risk factor for arterial embolic events? J Am Coll Cardiol. 1995;26:203-10. 
2. Orbison JL. Thrombosis of anomalous chordae in the right atrium (Chiari's network). Am Heart J. 1949;37:119-22.

3. Edwards P, Wozniak M, Corretti M, Price TR. Cardiac Chiari network as an etiology for embolic stroke. J Stroke Cerebrovasc Dis. 1994;4: 238-41.
4. Schwimmer-Okike N, Niebuhr J, Schramek GG, Frantz S, Kielstein H. The presence of a large Chiari network in a patient with atrial fibrillation and stroke. Case Rep Cardiol. 2016;2016:4839315.

5. Islam AKMM, Sayami LA, Zaman S. Chiari network: a case report and brief overview. J Saudi Heart Assoc. 2013;25:225-9. 